\title{
A Novel Path Control Algorithm for Networked Underwater Robot
}

\author{
Minghong She $\mathbb{C D}^{1,2}$ and Liyu Tian ${ }^{2}$ \\ ${ }^{1}$ School of Automation, Chongqing University, Chongqing, China \\ ${ }^{2}$ Computer College, Chongqing College of Electronic Engineering, Chongqing, China
}

Correspondence should be addressed to Minghong She; mh_she@126.com

Received 13 December 2017; Accepted 20 May 2018; Published 2 July 2018

Academic Editor: Keigo Watanabe

Copyright (C) 2018 Minghong She and Liyu Tian. This is an open access article distributed under the Creative Commons Attribution License, which permits unrestricted use, distribution, and reproduction in any medium, provided the original work is properly cited.

Under the network environment, the traditional control method of underwater Robot path has the disadvantages of low control accuracy, large error, and inefficiency. This paper proposes a novel path control method for underwater Robots based on the NURBS (nonuniform rational B-spline, NURBS) curve fitting method, which utilizes a sensor or camera to detect the static and dynamic obstacles, establishes the kinematics model of underwater Robots, gets the target function of rob shortest path, and analyzes underwater Robot constraints. According to the basic fluid mechanics, the resistance of the underwater Robot is determined. The filter function is used to smooth the process, and the NURBS curve fitting method is applied to control the path of the underwater Robot. Experimental results show that the improved method that proved to be practical is superior to the traditional one in the aspect of control time and accuracy.

\section{Introduction}

With the depletion of land resources, money, time, and manpower are invested to develop underwater resources [1]. Therefore, underwater Robots are a concern for people of many countries [2]. In order to complete an underwater task for one time, a variety of underwater Robots complex operation and safe navigation are to be carried out in accordance with the corresponding navigation path $[3,4]$.

To achieve the best navigation plan and keep the underwater Robot running on the scheduled course [5], it is necessary to ensure the maneuverability of the underwater Robot $[6,7]$. When the underwater Robot has good handling performance, it cannot only maintain stable driving course, depth, and speed but also quickly change the heading, depth, and speed and correctly perform all kinds of operations [8]. The path control of underwater Robot affects the performance of underwater Robot [9]. The method of networking can effectively control the running path of underwater Robots and shorten the control time [10, 11]. Therefore, the underwater Robot path control under the networked environment has become the focus of research in this field and has attracted the attention of many scholars.

Mahmoodabadi, Mohammad Javad et al. [12] mainly aims at controlling the path of underwater Robot when the underwater Robot is avoiding the obstacle and the direction of movement is not corrected. Based on the establishment of integrated navigation model and the detection based dynamic obstacle avoidance strategy, the discrete time multithread concurrent simulation framework and model are used to design the effective control of underwater Robot path. However, the method wastes much time. Zanoli, Silvia M., and Giuseppe Conte [13] aim at resolving the problem of low control speed or zero speed state in traditional control method, and the frame control moment gyroscope is introduced as the path control executive mechanism to realize the path control of the underwater Robot. This method can effectively shorten the control time of the path, but with a problem of low control accuracy [14]

To solve the above problems, a path control method of underwater Robot based on NURBS curve fitting method is proposed. The experimental results show that the control accuracy and efficiency are improved by adopting the presented control method. 


\section{Construction of Kinematics Model of Underwater Robot}

When the underwater Robot is effectively controlled under the condition of network, it is necessary to have a good understanding of the underwater Robot. The use of sensors or cameras to detect static and dynamic obstacles and the establishment of underwater Robot kinematics model can improve the path control method to provide the basis.

When the kinematic model of the underwater Robot is established, the head adjustment of the target underwater Robot can be obtained by adjusting the amount of integration processing [15]. The kinematic model is given by

$$
H_{g 1}=\sum K_{r 2} C_{r}
$$

where $C_{r}$ is output value and $K_{r 2}$ is weighted coefficient.

For the existence of the interference of underwater Robots, the use of arithmetic mean filtering method for filtering to eliminate the Gaussian noise has a positive effect [16]. When using the most single arithmetic mean filter, the data received at each time is replaced by the average of the received data in the neighborhood of the length of time. The expression is

$$
y(n)=\frac{1}{m} \sum_{i=1}^{m} x(n-i)
$$

where $m$ is constant, $i$ is the number of filters, and $x(n-i)$ is the wavelength of $n-i$ filter.

In the filter, the high frequency of interference removal effect will not achieve the best results. In this case, it is necessary to suppress a higher frequency of interference by adding a virtual damping force $[17,18]$. This makes the response of the underwater Robot relatively slow, increasing its stability. The expression is

$$
u_{i}=\frac{\Delta t e_{i}+T u_{i-1}}{\Delta t+T}
$$

where $\Delta t$ is the sampling period, $T$ is the inertia constant, $e_{i}$ is the time deviation of $i$, and $u_{i-1}$ is the response time of time $i$.

In the case of satisfying the absolute stability of the underwater Robot, the positive moment is used as the stability storage device [18] and then the balance coefficient can be changed as follows:

$$
C_{v}=1-\frac{M_{w}\left(m^{\prime}+Z_{q}^{\prime}\right)}{M_{q}^{\prime} Z_{w}^{\prime}}
$$

During the operation of the underwater Robot, the obstacle in the underwater environment will form a certain potential field and the repulsion force of $F_{r e p}$ is generated for the underwater Robot. The closer the distance is, the larger $F_{\text {rep }}$ will; that is to say, the closer the underwater Robot and the obstacle, the greater the potential energy of the underwater Robot and vice versa [19]. This potential field is similar to the potential field, inversely proportional to the distance, so the repulsive potential function can be expressed as

$$
\begin{gathered}
\frac{K_{r}}{\rho\left(X, X_{o b s}\right)} \quad \rho \leq \rho_{m} \\
0 \quad \rho \prec \rho_{m}
\end{gathered}
$$

where $K_{r}$ is the repulsive potential field constant, $X$ is the position vector of the underwater Robot, $X_{o b s}$ is the obstacle position vector in the water, $\rho$ is the shortest distance between the underwater Robot and the obstacle, and $\rho_{m}$ is the range of the repulsive potential field. When the distance between the underwater Robot and the obstacle is greater than $\rho_{m}$, the repulsive potential field does not interfere with the operation of the underwater Robot.

To avoid the collision between the underwater Robot and the obstacle, it is required to set a minimum safe distance $\rho_{0}$, when $\rho \rightarrow \rho_{0}$ and $F_{r e p} \rightarrow \infty$, and to ensure that $F_{r e p}$ is continuous, the expression of $F_{r e p}$ is

$$
\begin{aligned}
& F_{\text {rep }} \\
& \quad= \begin{cases}K_{r}\left(\frac{1}{\left(\rho\left(X, X_{\text {obs }}\right)-\rho_{0}\right)^{2}}-\frac{1}{\left(\rho_{m}-\rho_{0}\right)^{2}}\right) & \rho \leq \rho_{m} \\
0 & \rho>\rho_{m}\end{cases}
\end{aligned}
$$

Based on the above, sensors or cameras are used to monitor static and dynamic underwater obstacles and to obtain the position, speed, and acceleration information of obstacles in real time. These messages can fuse into moving obstacles. A number of navigation paths represented by time $t$ can be used in the obstacle motion constraints of underwater Robots. Suppose that $o b s_{k}$ is a rigid body with a center of $\alpha_{k}(t)=\left(\alpha_{1 k}(t), \alpha_{2 k}(t)\right), k=1,2, \ldots, q, t \in\left[0, t_{k}\right]$ and $\alpha_{k}()=$. $1,2, \ldots, q$ is a continuous real vector valued function, also the path of motion obstacles. Suppose that the underwater Robot and obstacle $k$ can be represented by $T_{\text {rob }}$ and $T_{k}$, respectively, $k=1,2, \ldots, q$. When the constraint of the underwater Robot is $\dot{x}_{1} \sin \theta-\dot{x}_{2} \cos \theta=0$ without lateral collision and the general coordinate system is $q(t)=\left[x_{1}(t) x_{2}(t) \theta(t)\right]^{T}$, the kinematic model of the underwater Robot is expressed as follows:

$$
q=\left[\begin{array}{cc}
\cos \theta t & 0 \\
\sin \theta t & 0 \\
0 & 0
\end{array}\right]\left[\begin{array}{c}
v(t) \\
w(t)
\end{array}\right]
$$

where $v(t)$ and $\omega(t)$ are, respectively, tangential velocity and angular velocity. In the navigation process, the speed of underwater Robot should be regulated within the maximum allowable speed to prevent the underwater Robot from colliding; meanwhile the underwater Robot must follow the rules of acceleration. 


\section{Improvement of Path Control Method for Underwater Robot}

3.1. Constraint Analysis. According to the kinematic model of the underwater Robot, the objective function of minimizing the path length is obtained:

$$
f(h)=\min \sum \frac{h\left(2 \sum_{f=1}^{F} E(f h)-E(0)-E(t f)\right)}{2}
$$

On this basis, various constraints are introduced into the objective function as the penalty term and the expression of the self-adaptation function is obtained:

$$
\begin{aligned}
\min F_{p c n a}= & \sum \frac{h\left(2 \sum_{f=1}^{F} E(f h)-E(0)-E(t f)\right)}{2} \\
& +c_{n} \sum_{f=0}^{F} \max \left(0, X^{l}-E\left(t^{\prime}\right)\right)
\end{aligned}
$$

where $t^{\prime}=f h, c_{n}$ is a larger value that is penalized for breaking the constraint, $n=1, \ldots, 9 ; X^{l}$ is the boundary area of its rob activity. Their constraint types are as follows:

(i) Boundary Constraint. Assuming that $X$ is the boundary of the selected area, the underwater Robot should be subject to the following motion boundary constraints during the moving process:

$$
\begin{aligned}
p(0) & =\left(x_{0}, \theta_{0}\right) \\
p\left(t_{f}\right) & =\left(x_{f}, \theta_{f}\right) \\
X_{0}, X_{f} & \in X
\end{aligned}
$$

(ii) Movement Restriction. In the process of underwater Robot navigation, there is a certain motion constraint. When the ship is sailing along the planning path, its acceleration and speed are prevented from colliding in the prescribed area. In literature [20], its motion constraint is expressed as follows:

$$
\begin{aligned}
v(t) & \geq v_{\min } \\
v(t) & \leq v_{\max } \\
\omega(t) & \geq \omega_{\min } \\
\omega(t) \leq \omega_{\max } & \\
& t \in\left[0, t_{f}\right]
\end{aligned}
$$

(iii) Obstacle Avoidance Constraint. To ensure that underwater Robots maintain a minimum safe distance from other static or moving underwater obstacles during navigation, $d_{\text {orb } k}$ and $d_{o k}$ are setting as the given safety distance and actual distance of $r o b$ and $\alpha_{k}$, respectively; therefore, $d_{\text {orb } \_k}$ is expressed as

$$
d_{o r b \downarrow}=\left\|x(t)-\alpha_{k}(t)\right\|_{2}-\left(r+r_{k}\right)
$$

where $1 \leq k \leq q$. Thus, in $X$, rob should satisfy the following barrier constraints:

$$
\begin{aligned}
& d_{\text {obs } \_}-d_{\text {ok }}(t) \leq 0, \\
& \forall k=1, \ldots, q . t \in\left[0, \max \left(t_{f}, t_{k}\right)\right]
\end{aligned}
$$

3.2. Implementation of the Proposed Method. Based on the analysis of constraints for underwater Robot and the basic fluid mechanics, the resistance of underwater Robot is determined, the filter functions are smoothed, and underwater Robot path is controlled by using NURBS curve fitting method

In view of the basic fluid dynamics, the total resistance $R_{t}$ of the underwater Robot can be divided into frictional resistance $R_{f}$ and residual resistance $R_{r}$. When the underwater Robot is in water for a long period of time, the wave resistance is negligible and the resistance is as follows:

$$
R_{m}=0.5 \rho V_{s}^{2} S\left(C_{f}+\Delta C_{f}+C_{p v}\right)
$$

where $\rho$ is the density of water, $V_{s}$ is the speed of underwater Robot, $S$ is wet surface area, $C_{f}$ is friction resistance coefficient, $\Delta C_{f}$ is friction resistance subsidy coefficient, and $C_{p v}$ is viscosity resistance coefficient.

Supposing that the running path of an underwater Robot can be represented by a weighted directed graph $G(V,\{E\})$, in which the set $V$ is a set of fixed points, $n$ is the fixedpoint number of the fixed-point set, $E$ is the arc set of the $m$ arc, $\left\langle v_{i}, v_{j}\right\rangle$ is the arc from $v_{i}$ to $v_{j}$ in $E$, and $d\left\langle v_{i}, v_{j}\right\rangle$ is the nonnegative weight of the $\operatorname{arc}\left\langle v_{i}, v_{j}\right\rangle$. Assuming that $v_{i}$ and $v_{j}$ are the paths from vertex $v_{i}$ to $v_{j}$ in $V$, the expression is as follows:

$$
P=\left\{v_{0}=v_{i}, \ldots, v_{n}=v_{j}\right\}
$$

For a path sequence consisting of $n$ path points, the length of the $\left[p_{0}, p_{1}, \ldots, p_{n-1}, p_{n}\right]$ path is

$$
L_{n}=\sum_{k=1}^{n} d_{p_{k-1}, p_{k}}
$$

where $d_{p_{k-1}, p_{k}}$ is the straight-line distance of the $p_{k-1}$ and $p_{k}$ path segments. The evaluation function is $f_{L}=L_{n} / d_{\text {min }}$, including the fact that $d_{\text {min }}$ is the linear length of $p_{0}$ and $p_{n}$. When $f_{L}=1$, the path length is the shortest; that is, the underwater Robot can sail straight from $p_{0}$ to $p_{n}$. Considering the operating state of underwater Robot as a discrete process, the position of some obstacles will change, causing it to be controlled in the wrong direction. So the filtering function is used to smooth the filtering function:

$$
h_{k}=\frac{h_{k-l}+2 h_{k-l+1}+\cdots+2 h_{k+l-1}}{2 l+1}
$$

where $h_{k}$ is the density of obstacles after smoothing and $l$ is the filter length.

It is assumed that the entire underwater Robot path using the DPSS algorithm is $D=\left\{d_{1}, d_{2}, \ldots, d_{n}\right\}$, including the fact 


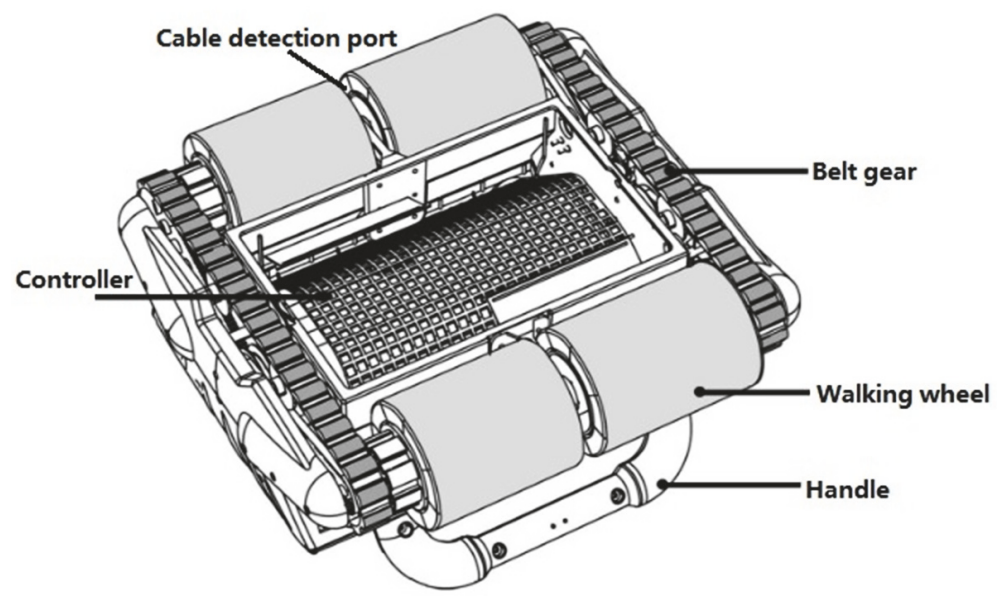

FIgURE 1: The structure of the cable detection underwater Robot.

that $d_{n}$ is the $n$ obstacle node on the path, and the Euclidean distance between all two adjacent obstacles is defined as

$$
L=\sum_{n=1}^{l} \sqrt{\left(x_{n+1}-x_{n}\right)^{2}+\left(y_{n+1}-y_{n}\right)^{2}}
$$

where $\left(x_{n}, y_{n}\right)$ is the coordinates of the grid node $d_{n}$.

The minimum turning radius $R_{\min }$ is the limit condition which cannot be ignored in underwater Robot path planning. Path control adopts the NURBS curve fitting method to determine the minimum turning radius of the underwater Robot, so as to realize the rapid and safe navigation of the underwater Robot in water. Expression is as follows:

$$
f_{R}= \begin{cases}\sum_{k=1}^{n} \frac{1}{\left(r_{k}-R_{\min }\right)}, & r_{k} \geq R_{\min } \\ \infty, & r_{k} \prec R_{\min }\end{cases}
$$

In (19), $r_{k}$ is the turning radius of the path $k$ point. When $r_{k} \prec$ $R_{\min }$, the path control caused by the turning is infinite, not worth the candle.

In order to satisfy the minimum turning radius of an underwater Robot, the maximum curvature $k_{\max }$ of the optimal path generated must satisfy the following constraints:

$$
k_{\max } \prec \frac{1}{R_{\min }}
$$

For the underwater Robots with different running paths, it is possible to satisfy the minimum turning radius requirement of the underwater Robot by selecting the appropriate parameter $R$ to set up the upper model. The path control method obtained from the previous analysis is in accord with the control characteristics and motion characteristics of the underwater Robot.

\section{Simulation Results}

In order to verify the effectiveness and feasibility of the improved control method, it is necessary to make a comparative analysis of the improved methods. The experiment

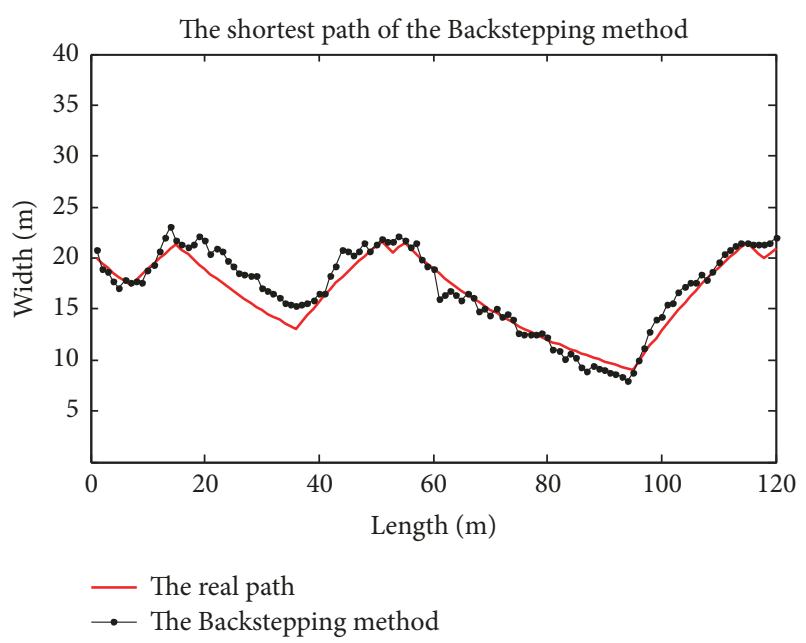

FIGURE 2: The shortest path of the Backstepping method.

is simulated by MATLAB, and the result is compared by NURBS curve fitting method. The simulated cable detection underwater Robot is shown in Figure 1. The hardware test platform is under the condition of single-phase inverter unit and controlled rectifier of model PM201CL1A061. Platform identification algorithm is DSP27334 main control chip. The production company is TI.

4.1. Navigation Path Comparison. In order to verify the effectiveness of the improved method, the experimental range is set at a certain number of underwater faults in $100 * 40$. Backstepping method, immune fuzzy PID method, and improved method are adopted to control the shortest path, respectively. The results are shown in Figures 2, 3, 4, and 5.

In Figure 2, the black dotted line represents the control point of the underwater Robot Backstepping method in the case of underwater obstacles.

In Figure 3, the blue cross line represents the control point of the underwater Robot immune fuzzy PID method in the case of underwater obstacles. 


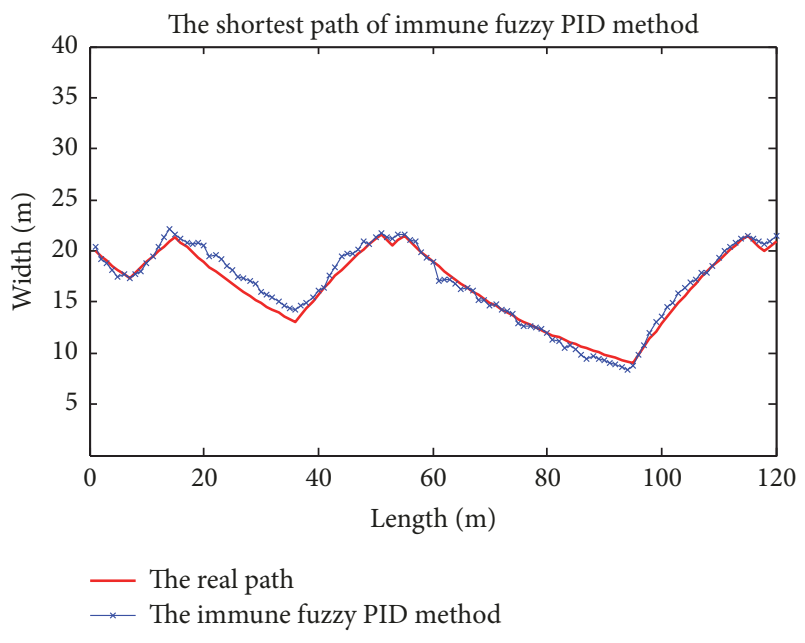

FIGURE 3: The shortest path of immune fuzzy PID method.

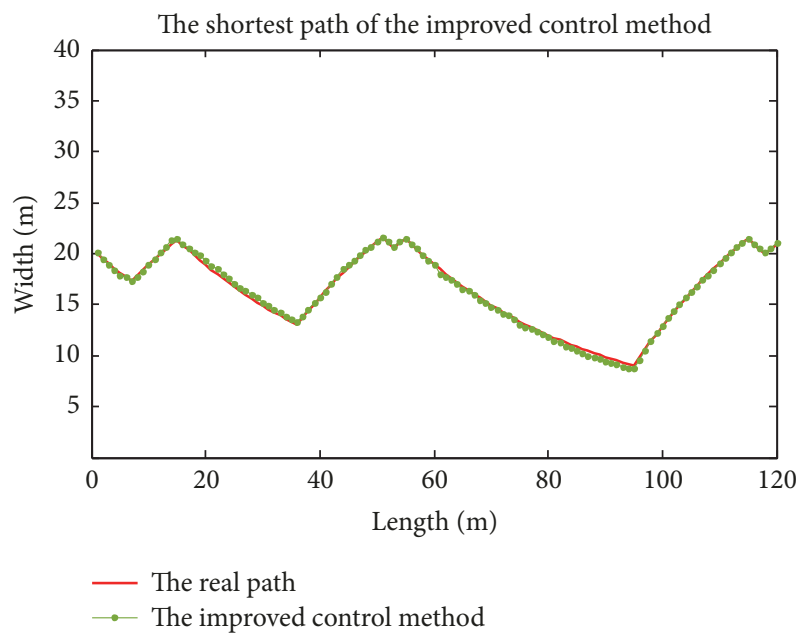

FIGURE 4: The shortest path of the improved control method.

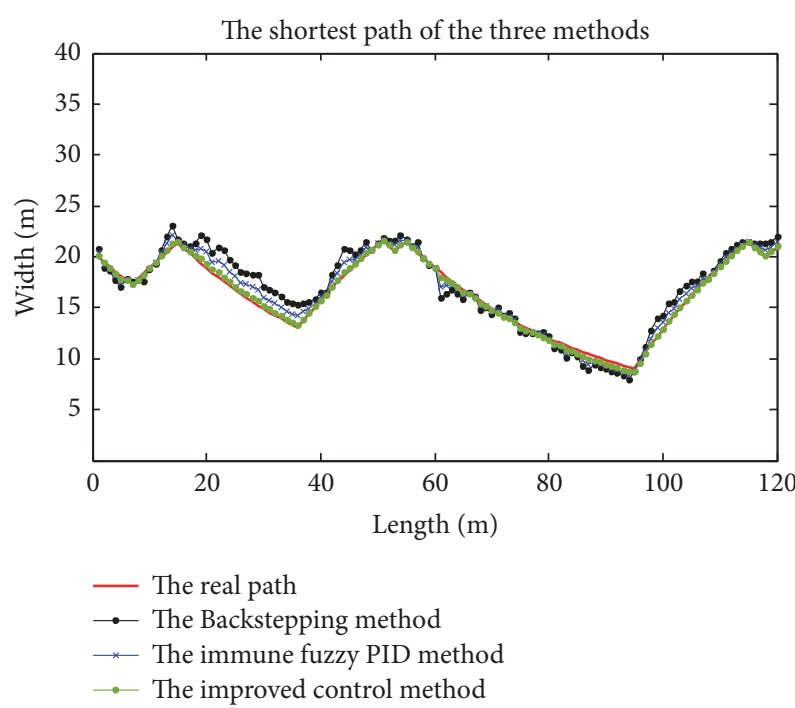

FIGURE 5: The comparison of the shortest paths of the three-control methods.

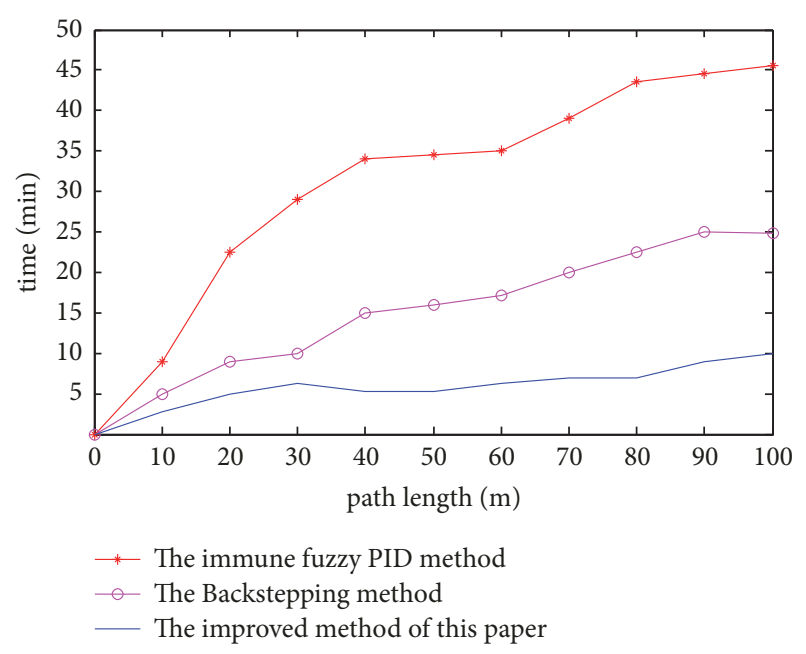

Figure 6: Time comparison analysis.

The light green dotted line in Figure 4 shows the control point of the underwater Robot of the improved control method in the case of underwater obstacles.

In Figure 5 shows the result of the comparison of the shortest paths of the three-control methods.

As can be seen from Figures 2-5, the results of three methods are different under the condition of underwater Robot that has a certain range of motion. The Backstepping method was controlled 62 times, of which only 29 were on the best path for the underwater Robot. When the obstacles in the water increase, the trajectory of the Robot deviates from the optimal path and the control is not effective enough. The immune fuzzy PID method increases the number of control accordingly, and the control increases to 85 times with the increase of the obstacles in water. The path of navigation deviates from the optimum path, only 4 times returning to the best path, but rapidly deviating. This shows that the stability is poor; the improved method can make the number of control 100 times; the whole path of the underwater Robot deviates little from the optimal path; and it coincides with the optimal path many times. It shows good stability. From this we can see that the presented method has certain advantages.

4.2. Complexity Analysis. In the optimal path control method of underwater Robot, the degree of underwater Robot path control is described by the running time of underwater Robot under the same path of the same length. This quantitative description of underwater Robot navigation path control method is highly operational and can be used to determine the trajectory control of underwater Robot directly. As a result, the time of the voyage can be calculated directly by the length of the section and the speed of sailing. The expression for travel time is

$$
\text { Rtime }=\frac{\text { Rdist }}{\text { Rspace }}
$$

Rdist is the length of the current section, and Rspace is the average speed of the underwater Robot. The time required for the Robot to run in three ways is shown in Figure 6. 
As you can see from Figure 6, when the path length is constant, the three methods vary in time. By using the Backstepping method, the running time is about $21.89 \mathrm{~min}$ and the time spent in $0-20 \mathrm{M}$ is rapidly increasing while the time spent in 20-30 does not increase. After 30m, it had been on the rise and had no tendency to decline. The immune fuzzy PID method takes about 32.5 min time and takes a long time. As the path of the ship increases, the time of sailing increases accordingly. Only when the region is stable at $40-60 \mathrm{~m}$ does the rest of the road become on the rise. The time taken by the improved method is about $4.98 \mathrm{~min}$, and the running time increases with the increase of path length, but the rising trend is not significant. This method has good stability and has some advantages.

\section{Concluding Remarks}

The traditional control methods always have the problems of poor effect, nonfixed path selection, and long time consuming. Hence, a path control method for underwater Robots based on NURBS curve fitting method is proposed. Experimental comparison results are illustrated:

(i) Although the number of control is greater than the Backstepping method and the immune fuzzy PID method when navigating in the same path length area, the overall control is better than the Backstepping and the immune fuzzy PID method.

(ii) Compared with the Backstepping and the immune fuzzy PID method, the navigation time of the improved method is shortened by $16.91 \mathrm{~min}$ and $27.52 \mathrm{~min}$, respectively, with good stability and advantages.

\section{Conflicts of Interest}

The authors declare that they have no conflicts of interest.

\section{Acknowledgments}

This work is supported by the Science and Technology Research Program of Chongqing Municipal Education Commission (Grant no. KJ1729403); the National Natural Science Foundation of China (no. 61403055); Chongqing Education Science and Planning Issues, project name: the Current Situation Analysis and Countermeasure Research of Modern Apprenticeship Practice in Vocational Colleges (no. 2017GX-422); the Fundamental Research Funds for the Central Universities (Grant no. CDJXS12 1711 01); Intelligent Robot Technology Research Center of Chongqing College of Electronic Engineering (no. XJPT201705).

\section{References}

[1] F. A. Azis, M. S. M. Aras, M. Z. A. Rashid, M. N. Othman, and S. S. Abdullah, "Problem identification for Underwater Remotely Operated Vehicle (ROV): A case study," in Proceedings of the 2nd International Symposium on Robotics and Intelligent Sensors 2012, IRIS 2012, pp. 554-560, mys, September 2012.

[2] W. Zheng, Q. Zou, and W. Ni, "Design and application of underwater unmanned vehicle simulation system for navigation and obstacle avoidance," Xitong Fangzhen Xuebao / Journal of System Simulation, vol. 28, no. 1, pp. 91-98, 2016.

[3] L. Li, X. Yibo, L. Li, and et al., "Path control of submarine swing arm tracked mobile platform based on Backstepping method," Mining and Metallurgy Engineering, vol. 35, no. 6, pp. 1-5, 2015.

[4] Z. Chaohua, T. Guoyuan, H. Daomin, and et al., "Design of DSP control system for attitude control moment gyros group of autonomous underwater Robot," China Ship Research, vol. 11, no. 5, pp. 107-112, 2016.

[5] O. Cerman and P. Hušek, "Adaptive fuzzy sliding mode control for electro-hydraulic servo mechanism," Expert Systems with Applications, vol. 39, no. 11, pp. 10269-10277, 2012.

[6] M. J. Mahmoodabadi, M. Taherkhorsandi, and A. Bagheri, "Optimal robust sliding mode tracking control of a biped robot based on ingenious multi-objective PSO," Neurocomputing, vol. 124, pp. 194-209, 2014.

[7] A. Bagheri, T. Karimi, and N. Amanifard, "Tracking performance control of a cable communicated underwater vehicle using adaptive neural network controllers," Applied Soft Computing, vol. 10, no. 3, pp. 908-918, 2010.

[8] S.-Y. Li, C.-H. Yang, C.-T. Lin, L.-W. Ko, and T.-T. Chiu, "Adaptive synchronization of chaotic systems with unknown parameters via new backstepping strategy," Nonlinear Dynamics, vol. 70, no. 3, pp. 2129-2143, 2012.

[9] J. Javadi Moghaddam, M. H. Farahani, and N. Amanifard, "A neural network-based sliding-mode control for rotating stall and surge in axial compressors," Applied Soft Computing, vol. 11, no. 1, pp. 1036-1043, 2011.

[10] M. J. Mahmoodabadi, S. Momennejad, and A. Bagheri, "Online optimal decoupled sliding mode control based on moving least squares and particle swarm optimization," Information Sciences, vol. 268, pp. 342-356, 2014.

[11] M. J. Mahmoodabadi, S. Arabani Mostaghim, A. Bagheri, and N. Nariman-zadeh, "Pareto optimal design of the decoupled sliding mode controller for an inverted pendulum system and its stability simulation via Java programming," Mathematical and Computer Modelling, vol. 57, no. 5-6, pp. 1070-1082, 2013.

[12] J. J. Valera García, V. Gómez Garay, E. Irigoyen Gordo, F. Artaza Fano, and M. Larrea Sukia, "Intelligent Multi-Objective Nonlinear Model Predictive Control (iMO-NMPC): Towards the 'on-line' optimization of highly complex control problems," Expert Systems with Applications, vol. 39, no. 7, pp. 6527-6540, 2012.

[13] S. M. Zanoli and G. Conte, "Remotely operated vehicle depth control," Control Engineering Practice, vol. 11, no. 4, pp. 453-459, 2003.

[14] J. Javadi-Moghaddam and A. Bagheri, "An adaptive neurofuzzy sliding mode based genetic algorithm control system for under water remotely operated vehicle," Expert Systems with Applications, vol. 37, no. 1, pp. 647-660, 2010.

[15] B. W. Hobson, A. D. Sherman, and P. R. McGill, "Imaging and sampling beneath free-drifting icebergs with a remotely operated vehicle," Deep-Sea Research Part II: Topical Studies in Oceanography, vol. 58, no. 11-12, pp. 1311-1317, 2011.

[16] Z. Chaohua, T. Guoyuan, T. Daomin et al., "Design of DSP control system for attitude control moment gyros group of autonomous underwater Robot," China Ship Research, vol. 11, no. 5, pp. 107-112, 2016.

[17] Z. Yang, F. Liu, and Y. Wang, "Path following of underactuated surface vessels based on neural sliding mode," Ship Building of China, vol. 56, no. 2, pp. 45-55, 2015. 
[18] W. Yanqiong and C. Shiping, "Dual path routing admission control protocol for smart road wireless networks," Computer Engineering, vol. 41, no. 11, pp. 24-29, 2015.

[19] S. Wei, H. Yulong, and L. Baoshan, "Non symmetric unmanned amphibious platform path tracking control," Acta Armamentarii, vol. 37, no. 7, pp. 1161-1169, 2016.

[20] Z. Chu, D. Zhu, and S. X. Yang, "Observer-based adaptive neural network trajectory tracking control for remotely operated vehicle," IEEE Transactions on Neural Networks and Learning Systems, vol. 28, no. 7, pp. 1633-1645, 2017. 


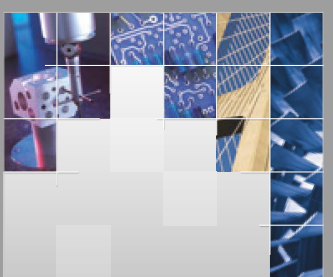

\section{Enfincering}
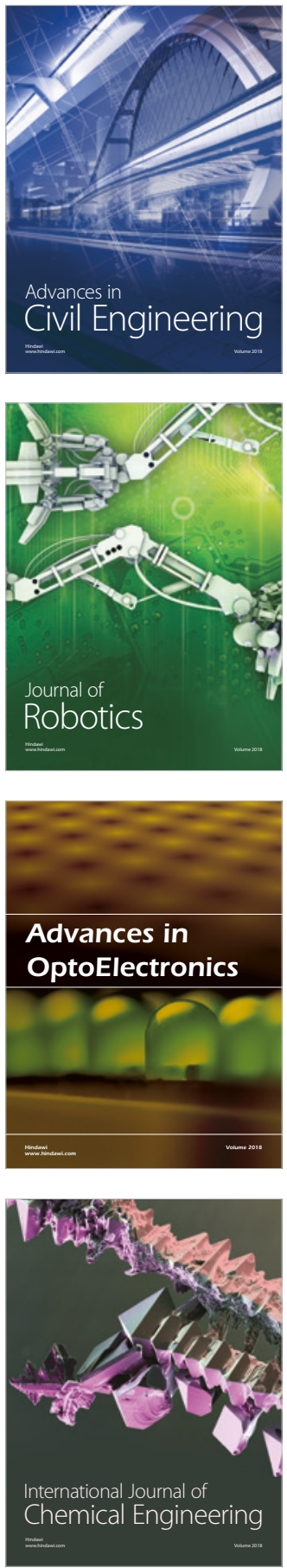

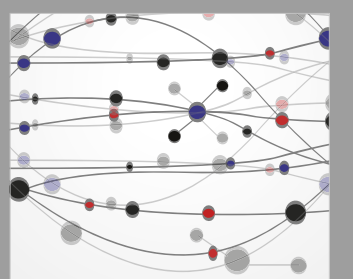

\section{Rotating \\ Machinery}

The Scientific World Journal

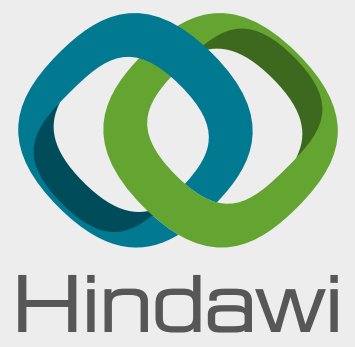

Submit your manuscripts at

www.hindawi.com
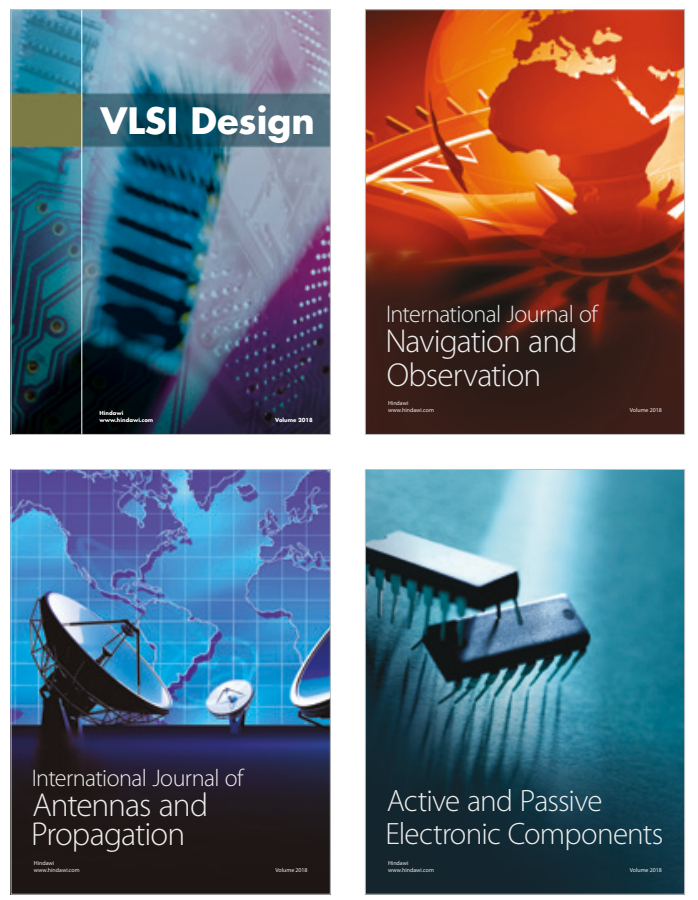
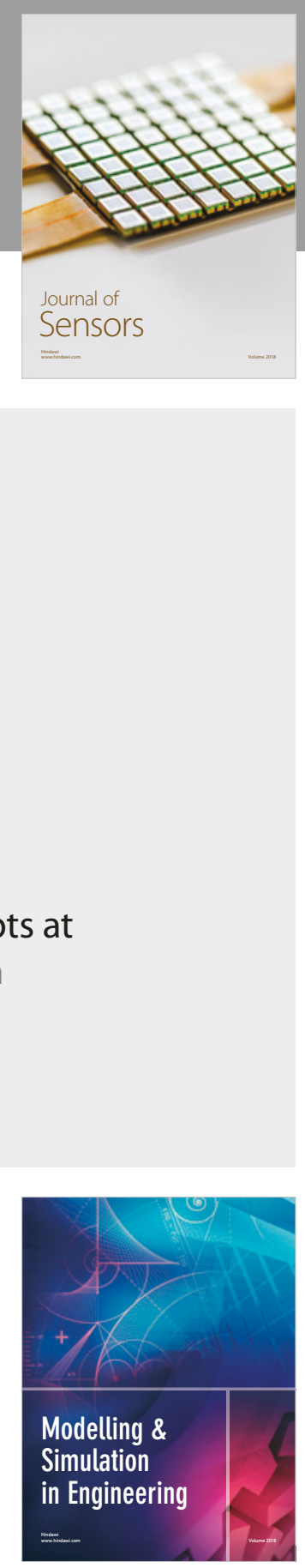

\section{Advances \\ Multimedia}
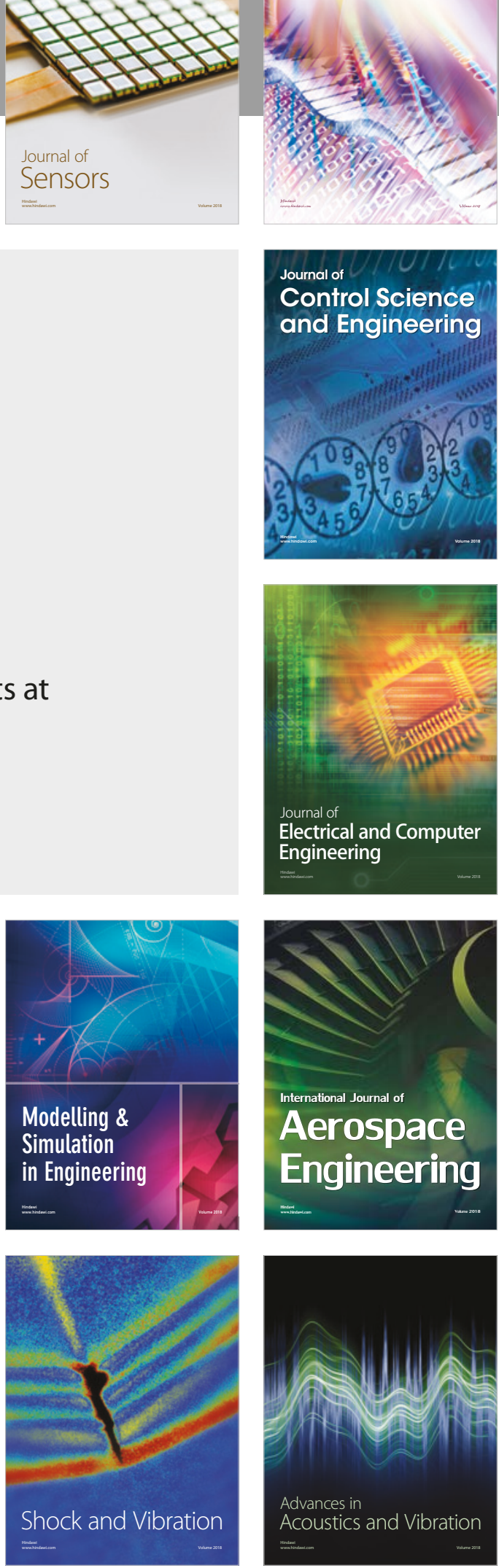